\title{
Maladaptive Bias for Extrahippocampal Navigation Strategies in Aging Humans
}

\author{
Jan M. Wiener, ${ }^{1}$ Olivier de Condappa, ${ }^{1}$ Mathew A. Harris, ${ }^{2}$ and Thomas Wolbers ${ }^{3,4}$ \\ ${ }^{1}$ Psychology Research Centre, Bournemouth University, Poole, BH12 5BB, United Kingdom, ${ }^{2}$ Centre for Cognitive and Neural Systems, University of \\ Edinburgh, Edinburgh, EH1 2QL, United Kingdom, ${ }^{3}$ German Center for Neurodegenerative Diseases (DZNE), Aging and Cognition Research Group, D- \\ 39120 Magdeburg, Germany, and ${ }^{4}$ Center for Behavioral Brain Sciences, D-39118 Magdeburg, Germany
}

Efficient spatial navigation requires not only accurate spatial knowledge but also the selection of appropriate strategies. Using a novel paradigm that allowed us to distinguish between beacon, associative cue, and place strategies, we investigated the effects of cognitive aging on the selection and adoption of navigation strategies in humans. Participants were required to rejoin a previously learned route encountered from an unfamiliar direction. Successful performance required the use of an allocentric place strategy, which was increasingly observed in young participants over six experimental sessions. In contrast, older participants, who were able to recall the route when approaching intersections from the same direction as during encoding, failed to use the correct place strategy when approaching intersections from novel directions. Instead, they continuously used a beacon strategy and showed no evidence of changing their behavior across the six sessions. Given that this bias was already apparent in the first experimental session, the inability to adopt the correct place strategy is not related to an inability to switch from a firmly established response strategy to an allocentric place strategy. Rather, and in line with previous research, age-related deficits in allocentric processing result in shifts in preferred navigation strategies and an overall bias for response strategies. The specific preference for a beacon strategy is discussed in the context of a possible dissociation between beacon-based and associative-cue-based response learning in the striatum, with the latter being more sensitive to age-related changes.

\section{Introduction}

Everyday navigation can be based on different strategies. The hippocampus plays a key role in cognitive map or place strategies that rely on allocentric processing, whereas the parietal cortex and striatal circuits are involved in route or response strategies (McDonald and White, 1994; Wolbers et al., 2004; Burgess, 2008). Aged rodents show deficits in tasks that rely on allocentric cues, whereas response strategies are less affected (Begega et al., 2001). Moreover, even when successfully trained on both place and response strategies, aged mice still show a preference for response strategies, suggesting an age-related increase in reliance on extrahippocampal systems to guide navigation (Nicolle et al., 2003).

Such age-related navigational impairments are likely to be related to the vulnerability of the hippocampus and surrounding structures to neurodegenerative processes (Lister and Barnes, 2009; Stranahan and Mattson, 2010). Given that similar physiological and morphological changes have been documented in ag-

Received Feb. 13, 2012; revised Jan. 31, 2013; accepted Feb. 11, 2013

Author contributions: J.M.W., 0.d.C., M.A.H., and T.W. designed research; J.M.W., 0.d.C., M.A.H., and T.W. performed research; J.M.W., 0.d.C., M.A.H., and T.W. analyzed data; J.M.W., 0.d.C., M.A.H., and T.W. wrote the paper.

We thank Jennifer Sturgess and Louis Chalmers for their help in data collection.

The authors declare no competing financial interests.

This article is freely available online through the J Neurosci Author Open Choice option.

Correspondence should be addressed to Jan M. Wiener, Bournemouth University, Psychology Research Centre, Fern Barrow, Poole, BH12 5BB, UK. E-mail: jwiener@bournemouth.ac.uk.

DOI:10.1523/JNEUROSCI.0717-12.2013

Copyright $\odot 2013$ the authors $\quad 0270-6474 / 13 / 336012-06 \$ 15.00 / 0$ ing humans (Raz et al., 2005, Yankner et al., 2008), it is not surprising that difficulties in using place strategies have been observed in older adults (Moffat and Resnick, 2002, Moffat et al., 2007, Iaria et al., 2009, Harris and Wolbers, 2012, Wiener et al., 2012). However, at present, the precise consequences of these changes in navigational computations are unknown.

In this study, we used a novel route-learning paradigm (1) to test whether cognitive aging does indeed lead to a shift in preferred navigation strategy and (2) to precisely identify the type of response strategy used by older adults. Response strategies are often conceptualized as a series of stimulus-response pairs in which landmarks serve as cues that become associated with motor responses defined relative to the body axis ("Turn left at the post office"; associative cue strategy). However, humans also use beacon-based strategies in which movement responses toward a landmark brings one closer to the goal but that do not require explicit encoding of directional information or motor responses ("Go toward the post office"; Waller and Lippa, 2007). Although both response strategies rely on striatal processing, some studies suggest a dissociation, with the dorsal and dorsolateral striatum implicated in associative-cue learning (Featherstone and McDonald, 2004, 2005) and the ventral and dorsomedial striatum involved in beacon-based strategies (Devan and White, 1999). Given that the dorsal striatum might be more sensitive to age-related neurodegeneration (Tupala et al., 2003), aged humans could be expected to show a preference for beacon-based over associative-cue-based strategy.

To test the hypothesis that cognitive aging not only results in a shift away from allocentric strategies but in a specific preference 

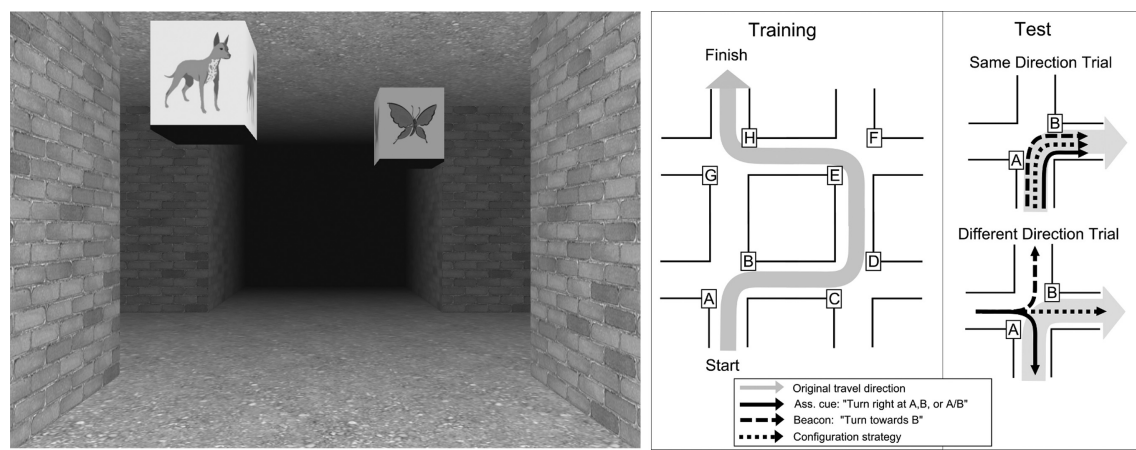

Figure 1. Left, Screenshot of one intersection; middle, schematic overview of the training route; right, two of the test trials. In same-direction trials, the intersections are approached from the same arm as during training: all strategies result in correct responses. In different-direction trials, the intersection is approached from an arm different from that of training. In the depicted example, the three strategies generate different responses; the associative cue strategy ["Turn right at A (or B)"] leads to a right turn, the beacon strategy ("Turn toward B") produces a left turn, whereas only the configuration strategy, relating the goal arm to the configuration of local landmarks, yields the correct response. Note that our definition of beacon strategy involves a turning response ("Turn toward"), whereas other definitions use more general responses ("Move toward"; Waller and Lippa, 2007). However, in the current paradigm, in which single landmarks are always adjacent to two arms, this would lead to ambiguity. Specifically, participants should also make "straight" responses on same-direction trials, which only happened in $3 \%$ of the same-direction trials, strongly suggesting that they adopted a "Turn toward" beacon strategy, thus eliminating any ambiguity.

for beacon-based strategies, we developed a novel experimental paradigm: participants first learned a route along a number of intersections and were then asked to rejoin the original route approaching the intersections from different directions. Trials in which participants approached the intersections from a direction different from that during training (see Fig. 1) allowed us (1) to compare the use and adoption of route-learning strategies between young and older participants and (2) to test for specific preferences for beacon-based strategies in older participants.

\section{Materials and Methods}

Vizard 3.0 (WorldViz) was used to create the virtual environment and the route-learning task, which were presented on a 24 -inch widescreen computer monitor.

Participants. Forty-seven participants [23 younger ( 12 females; mean age, 20.8 years); 24 older ( 12 females; mean age, 73.8 years)] took part in the experiment. The Montreal Cognitive Assessment (MoCA; Nasreddine et al., 2005) was administered to all participants to screen for mild cognitive impairment (MCI). One older participant was excluded based on the recently recommended MoCA cutoff score for MCI of 23 (Luis et al., 2009). During the analysis, one young and an additional six older participants were excluded because they did not successfully learn the route (for details, see Results). Mean age of the remaining 17 older participants (eight females) was $74.7 \pm 5.03$ years, and their mean MoCA score was $26.58 \pm 1.94$; the mean age of the remaining 22 younger participants (11 females) was $20.73 \pm 1.12$ years, and their mean MoCA score was $28.14 \pm 1.21$. Participants were paid $£ 6 / \mathrm{h}$ to partake in the study, which took 60-90 min to complete.

Procedure. Participants learned and were tested on one route that consisted of four four-way intersections (two left turns, two right turns; Fig. 1). Each of six experimental sessions involved a training phase and a subsequent test phase. During each training phase, participants were twice passively transported along the route (speed, $5 \mathrm{~m} / \mathrm{s}$ ) and were instructed to memorize the route. Each intersection along the route was characterized by two unique landmarks located in diagonally opposite corners, unambiguously identifying the intersection as well as the direction from which it was approached (Fig. 1). Including black fog in the environment ensured that only one intersection was visible at any time. In the test phase, participants were guided toward the intersections within the route and were asked to indicate the direction in which the original route proceeded by pressing the left, right, or up (i.e., straight) arrow key. Both responses and response times were recorded. In the test phase, each of the four intersections was either approached from the same arm as during training (resulting in four same-direction trials) or-excluding the arm where the route proceeded during trainingfrom the two remaining arms (resulting in eight different-direction trials), adding up to 12 trials per session. To avoid participants memorizing a series of movement directions during training (right-left-left-right), the 12 test trials were presented in randomized order. Crucially, same- and different-direction trials test different abilities: same-direction trials test participants' ability to successfully replicate the route, i.e., to replicate the direction changes experienced during the learning phase. These trials can therefore be considered a typical measure of route knowledge (Head and Isom, 2010) and can be solved using any of the three navigation strategies discussed above (beacon, associative cue, and configuration). In contrast, different-direction trials cannot be solved simply by associative cue-based or beaconbased strategies (Wiener et al., 2012). Instead, to unambiguously identify the direction in which the original route proceeded, these trials require allocentric place learning, i.e., the processing of the spatial configuration of local cues (landmarks) in relation to the goal arm. We refer to this allocentric place strategy as the configuration strategy. For two of the different-direction trials (see example depicted in Fig. 1), all three strategies produced a different response. By analyzing participants' responses for these different-direction trials for each of the six experimental blocks, we calculated the number of responses in line with each strategy, which allowed us to assess individual strategy preferences and changes thereof over the course of the experiment.

Before the experiment, participants were informed that they would approach the intersections from different directions and that they should respond as quickly and accurately as possible. To prevent learning from feedback, participants did not receive immediate feedback after making a decision in the test phase. However, repeated training sessions allowed participants to learn the spatial relationship of the local landmarks and the goal arm over the course of the experiment.

\section{Results}

Route-learning performance-i.e., the ability to repeat the learned route-was assessed in same-direction trials in which young participants performed better than older participants ( 81.13 vs $64.07 \%$ correct responses; $\left.t_{(35.64)}=3.01, p<0.01\right)$. The fact that variance in the older age group was higher than in the younger age group (Levene's test, $F_{(44)}=6.19, p=0.02$ ) suggests that some of the older participants found the task hard, whereas others performed well. Using $\chi^{2}$ tests, we tested whether individual participants' performance for same-direction trials significantly exceeded chance-level performance (with three possible movement directions, chance level was $33 \%$ or 8 of 24 samedirection trials). In fact, performance for six older participants did not exceed chance level, whereas performance of only one younger participant did not exceed chance level. Given that the aim of this study was the investigation of aging-induced shifts in route-learning strategies and the identification of the exact type of strategy used, we excluded these participants because they did not successfully learn the routes in the first place.

Because participants were selected based on their performance on the same-direction trials, included participants in both age groups were able to learn the route (Fig. 2). However, the older participant group showed specific deficits when approaching an intersection from an arm different from that experienced during encoding (Fig. 2). A general linear model with age (young, 
old) and gender as between-group factors and approach direction (same, different) and session (1-6) as within-subjects factors revealed that younger participants performed better than older participants (main effect of age, $F_{(1)}=20.601, p<$ $0.001)$, that performance was better when approaching intersections from the same direction as during training than from a different direction (main effect of approach direction, $F_{(1)}=142.49, p<$ 0.001 ), and that performance increased across sessions (main effect of session, $\left.F_{(5)}=18.981, p<0.001\right)$. In contrast, we did not observe a significant effect of gen$\operatorname{der}\left(F_{(1)}=0.012, p=0.91\right)$.

Of the two-way interactions, the session $\times$ age $\left(F_{(5)}=5.682, p<0.001\right)$ and the approach $\times$ age $\left(F_{(1)}=16.961, p<0.001\right)$ interactions were significant. Importantly, a significant three-way approach direction $\times$ session $\times$ age interaction $\left(F_{(5)}=5.356, p<0.001\right)$ suggested that both groups differed in how performance differences between same and different-direction trials evolved across the six sessions. Planned contrasts revealed that both groups showed a linear performance increase on same-direction trials (young, $F_{(35)}=16.56$, $p<0.001$; old, $F_{(35)}=7.675, p=0.009$ ), whereas only the young group showed this effect for the different-direction trials (young, $F_{(35)}=80.026, p<0.001$; old, $F_{(35)}=0.147, p=0.70$; interaction between linear increase and age, $\left.F_{(35)}=31.492, p<0.001\right)$. Together, these results demonstrate that performance differences between age groups cannot be explained by a nonspecific performance decrement or inattentiveness in the older group. Rather, older adults exhibited selective problems with using the correct allocentric place strategy throughout the experiment.

To assess strategy preferences, we calculated the percentage of responses in line with the three strategies (Fig. 2, right) for those different-direction trials that distinguished between all strategies (see example in Fig. 1). Because performance for differentdirection trials improved over experimental sessions for young participants, so did usage of the correct allocentric navigation strategy. In contrast, the older participant group showed very little change in strategy use over the six experimental sessions. To quantify strategy preferences and changes thereof, we ran separate ANOVAs for each of the three strategies with age (young, old) as a between-group factor and session (1-6) as a withinsubjects factor, and the percentage of trials the corresponding strategy was used as the dependent variable. Young participants relied more strongly on the configuration strategy than older participants $\left(F_{(1,37)}=38.42, p<0.001\right)$. The main effect of session $\left(F_{(5,37)}=15.32, p<0.001\right)$ was driven by the strong increase in configuration strategy use by the younger participants, which is reflected in the significant age $\times$ session interaction $\left(F_{(5,37)}=\right.$ 8.63, $p<0.001)$. The older participant group, compared with young participants, relied more strongly on the beacon strategy $\left(F_{(1,37)}=19.59, p<0.001\right)$. Although there was a significant main effect of session for beacon strategy usage $\left(F_{(3.62,133.90)}=\right.$ 2.99, $p=0.03)$, the interaction did not reach statistical significance $\left(F_{(3.62,133.90)}=2.37, p=0.06\right)$. The older participants also used the associative cue strategy more than the young participants $\left(F_{(1,37)}=6.30, p=0.02\right)$. However, usage of the associative cue strategy did not change over experimental sessions $\left(F_{(3.92,145.10)}=1.25, p=0.29\right)$, and there was no significant in- teraction between age group and session $\left(F_{(3.92,145.10)}=0.99\right.$, $p=0.41)$.

To investigate which of the two response strategies the older group preferred, we compared the percentage of responses in line with the beacon strategy with those in line with the associative cue strategy. This revealed that, as a group, older participants showed an overall preference for the beacon strategy $\left(t_{(16)}=3.54, p<\right.$ $0.01)$. This preference already existed in the first experimental session $\left(t_{(16)}=3.16, p<0.01\right)$ and did not change across the experiment (Fig. 2). Such a systematic preference for one strategy strongly suggests that the poor performance of the older group for different-direction trials was not unspecific or a result of different-direction trials being more difficult than the samedirection trials: in this case, we would have expected random behavior but no systematic bias.

Finally, the use of different navigation strategies by the two age groups should lead to a specific response time pattern: both the beacon and the associative cue strategy state that a turning response (beacon, "Turn toward ..."; associative cue, "Turn left/ right at ...") is activated on the recognition of the corresponding place or landmark. As long as the place is identified, this process is independent of approach direction. In contrast, the configuration strategy requires the arrangement of the landmarks at the intersections to be encoded. If a place is approached from a different direction, the observer needs to use the landmark configuration to determine their facing direction. In addition, computing the correct movement direction involves a second step: either the direct computation of the correct response from an allocentric representation of the intersection or a mental transformation of the observer's current viewpoint to match the one experienced during encoding. These additional processing steps should lead to an increase in response time when approaching intersections from a novel direction, which should be greater for the young because more participants in that group used the configuration strategy. In accordance with this prediction, a repeated-measures ANOVA not only revealed significant main effects of approach direction $\left(F_{(1,37)}=42.79, p<0.001\right)$ and age $\left(F_{(1,37)}=26.53, p=0.001\right)$ on response time but also a significant interaction between age group and approach direction $\left(F_{(1,37)}=\right.$ 9.80, $p<0.01$; Fig. 3). Post hoc $t$ tests revealed shorter response times for same-direction trials than different-direction trials for the young and old participant groups (young, $p<0.001$; old, $p=0.02$ ). Although it may seem surprising that overall older participants responded more quickly than younger participants, this can be explained by the larger proportion of 


\section{Response Time}
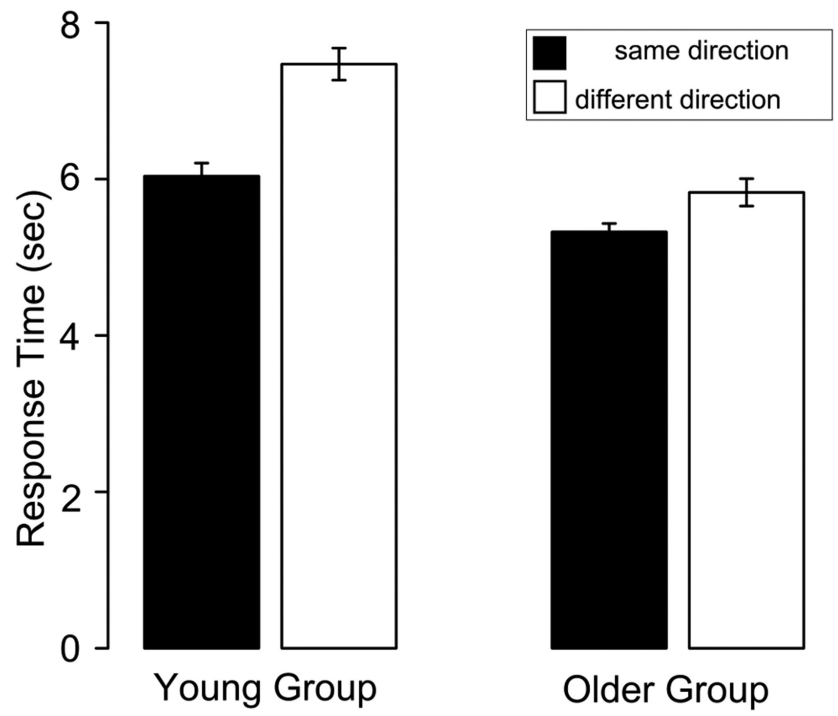

Figure 3. Average response times for same- and different-direction trials and both age groups. Data are collapsed across the experimental sessions (mean $\pm \mathrm{SE}$ ).

younger participants using the more time-consuming allocentric place strategy.

\section{Discussion}

We used a novel paradigm to investigate the effects of cognitive aging on the selection and adoption of navigation strategies. Our paradigm differs from other paradigms used in aging research, such as the (human) Morris water maze and the Y-maze (Rodgers et al. 2012), in a number of important aspects. First, participants learn a route consisting of multiple intersections, which makes it a somewhat more natural task. Second, our task not only allows for the investigation of initial strategy selection biases but also of shifts in bias, which are particularly sensitive measures in detecting age-related neurodegeneration (Nicolle et al., 2003). Finally, in contrast to previous studies (Rodgers et al., 2012) addressing strategy preferences in older adults, our paradigm allows distinguishing between associative cue-based and beacon-based response strategies.

In the experiment, participants were required to follow a previously learned route encountered from an unfamiliar direction, and, to do so successfully, they needed to use an allocentric place strategy. Our younger participants showed clear evidence for adopting this superior navigation strategy. In contrast, those older participants who were able to successfully recall the route when approaching intersections from the same direction as during training showed a consistent preference for a beacon-based strategy and were unable to use the appropriate place strategy, resulting in poor performance when rejoining the learned route from a novel direction. Given that allocentric spatial processing required for place strategies is thought to critically depend on hippocampal computations, we propose that age-related hippocampal degeneration (Raz et al., 2005) induced a shift in preferred navigation strategies toward beacon-based strategy in older participants.

In contrast to the rapid learning for the same-direction trials, initial performance in young participants was poor on trials when an intersection was approached from an unfamiliar direction. However, the subsequent improvement demonstrates that this group gradually developed an understanding of the spatial configuration of each intersection and the position of the goal arm, which enabled them to use the correct configuration strategy. This strategy might have involved additional mental transformations of one's viewpoint or a direct access of the allocentric location of the goal arm, both of which have been linked to observerindependent spatial computations in the hippocampal formation (King et al., 2002; Wolbers and Büchel, 2005).

Consistent with recent findings (Head and Isom, 2010), the old age group showed an overall route-learning deficit, i.e., when repeating the route as experienced during training. A higher performance variability across participants suggested that this effect was primarily driven by a subgroup who were unable to learn the route. These underperforming older participants were removed from the final dataset, after which both age groups performed well on same-direction trials, indicating similar route-learning abilities. However, in sharp contrast, the performance of the older group on the different-direction trials remained at chance throughout the experiment, demonstrating that even the older participants who reproduced the route successfully were unable to use a place strategy. Instead, they showed a preference for a beacon-based strategy and showed no evidence of changing their behavior across the six sessions.

Rodgers et al. (2012) recently showed a similar age-related bias for response strategies in a virtual Y-maze. Critically, the probe trial was administered after subjects had shown consistent performance over five learning trials. As a consequence, the response bias could be based on the suggested inability to use place strategies. However, given that age-related deficits in strategy switching are well established in various cognitive domains (Kray and Lindenberger, 2000), it could also reflect an inability to switch from a firmly established response strategy to a place strategy in the probe trial. In our study, we observed a bias for a beacon strategy from the first session onward, with very little change occurring over the course of the experiment. These results make the strategy switching account unlikely and point instead to an age-related deficit in processing allocentric cues.

Response strategies are thought to rely on striatal circuits, whereas allocentric processing relies heavily on spatial computations in the hippocampal formation (Hartley et al., 2003; Doeller et al., 2008). Critically, the hippocampus has been shown to take over route-based navigation when the caudate is dysfunctional (Voermans et al., 2004), but the striatum is unable to take over allocentric navigation after hippocampal lesions (GoodrichHunsaker et al., 2010). As a consequence, because the hippocampus is particularly sensitive to the deleterious effects of aging (Yankner et al., 2008), the preferred use of response strategies might reflect an inability to successfully compensate for impaired hippocampal computations that are necessary for processing allocentric cues. Instead, our older participants relied on navigational strategies mediated by extrahippocampal structures, which we presume include the striatum.

Going beyond a general bias toward response strategies, older adults showed a consistent preference for a beacon-based strategy over an associative-cue-based strategy. This specific preference might be related to a functional dissociation between the medial/ ventral and the lateral/dorsal striatum (Voorn et al., 2004). In rodents, the ventral and dorsomedial striatum receives extensive convergent input from multiple sensory and association areas of the neocortex and the limbic system, whereas the dorsal and dorsolateral striatum has a distinct topographic pattern of connections with somatosensory and motor areas (McGeorge and Faull, 1989). Similarly, in primates, the ventral striatum encompasses 
the medial wall of the caudate nucleus because of its inputs from regions such as the orbitofrontal cortex but not from primary or secondary motor cortices (Nakano et al., 2000; Haber, 2003). In contrast, the lateral part of the caudate receives cortical afferents from motor cortical areas as well as the somatosensory cortex. Hence, it is commonly thought of as part of the dorsal (or sensorimotor) striatum (Nakano et al., 2000). Although some functional neuroimaging studies point to a different homology (Balleine and O'Doherty, 2010), the patterns of connectivity indicate that the dorsal/dorsolateral striatum is more important for motor response learning as required for the associative cue strategy, whereas the ventral/dorsomedial striatum could be more important for beacon strategies.

Consistent with this anatomical dissociation, lesion studies in rodents implicate the dorsolateral striatum in stimulus-motor response learning (Packard and McGaugh 1996). Moreover, dorsomedial/ventral striatum lesions do not affect stimulus-motor response learning to the same extent as dorsal/dorsolateral lesions (Yin and Knowlton, 2004). Finally, dorsomedial lesions affect the learning of a beacon strategy, whereas dorsolateral lesions do not (Devan and White, 1999). However, these findings are reversed once the animal has been overtrained on the task, which suggests that the dorsomedial striatum might only be important for learning a beacon strategy, which was addressed in our experiment as well. In summary, there is ample evidence for a role of the dorsal/dorsolateral striatum in stimulus-motor response learning and somewhat weaker evidence for a role of the dorsomedial/ventral striatum in beacon-based navigation.

Given this anatomical and functional dissociation within the striatum, what could explain the bias for beacon over associative cue strategies in older adults? In humans, the caudate shows significant age-related neurodegeneration at similar rates compared with the hippocampus (Raz et al., 2005). Furthermore, dorsal striatal dopamine transporters and receptors appear to be more vulnerable to age-related decline than their ventral striatal counterparts (Tupala et al., 2003; Kim et al., 2011). Although we are not aware of any studies testing for a potential dissociation between the dorsolateral and dorsomedial striatum, we speculate that stimulus-motor response learning processes as required for the associative cue strategy might be similarly affected by aging as hippocampus-dependent place-based processes, whereas beacon-based navigation might be more resistant to age-related change.

To our knowledge, our study is the first to directly investigate the effects of cognitive aging on different response strategies. Older adults have been reported to be more likely to point out landmarks than turns as providing the most useful information for route navigation (Lipman, 1991). In addition, Alzheimer's disease patients appear to have intact representations of objects encountered during route learning, but they fail to integrate object and spatial information (Kessels et al., 2011). These findings are consistent with our findings, indicating that using landmarks as beacons - without the need to associate any directional/motor information-could be more resistant to age-related decline. This would also explain correlations between route-learning performance and changes in caudate volume during aging in routelearning paradigms that do not allow for beacon-based strategies (Head and Isom, 2010).

Together, we demonstrated an age-related deficit in allocentric processing, which may severely impair navigation when approaching a location on a known route from an unfamiliar direction. Importantly, of the egocentric response strategies that our novel task allowed us to study, older people showed a prefer- ence for the beacon strategy over the associative cue strategy. In support of animal findings, this preference points to a dissociation between beacon- and associative-cue-based learning in the striatum, with the latter being more sensitive to age-related changes. These findings not only provide additional insight into the navigational difficulties observed in normal cognitive aging, but they could also suggest directions for the development of novel navigational aids for elderly users.

\section{References}

Balleine BW, O'Doherty JP (2010) Human and rodent homologies in action control: corticostriatal determinants of goal-directed and habitual action. Neuropsychopharmacology 35:48-69. CrossRef Medline

Begega A, Cienfuegos S, Rubio S, Santín JL, Miranda R, Arias JL (2001) Effects of ageing on allocentric and egocentric spatial strategies in the Wistarrat. Behav Processes 53:75-85. CrossRef Medline

Burgess N (2008) Spatial cognition and the brain. Ann N Y Acad Sci 1124: 77-97. CrossRef Medline

Devan BD, White NM (1999) Parallel information processing in the dorsal striatum: relation to hippocampal function. J Neurosci 19:2789-2798. Medline

Doeller CF, King JA, Burgess N (2008) Parallel striatal and hippocampal systems for landmarks and boundaries in spatial memory. Proc Natl Acad Sci U S A 105:5915-5920. CrossRef Medline

Featherstone RE, McDonald RJ (2005) Lesions of the dorsolateral striatum impair the acquisition of a simplified stimulus-response dependent conditional discrimination task. Neuroscience 136:387-395. CrossRef Medline

Featherstone RE, McDonald RJ (2004) Dorsal striatum and stimulusresponse learning: lesions of the dorsolateral, but not dorsomedial, striatum impair acquisition of a simple discrimination task. Behav Brain Res 150:15-23. CrossRef Medline

Goodrich-Hunsaker NJ, Livingstone SA, Skelton RW, Hopkins RO (2010) Spatial deficits in a virtual water maze in amnesic participants with hippocampal damage. Hippocampus 20:481-491. CrossRef Medline

Haber SN (2003) The primate basal ganglia: parallel and integrative networks. J Chem Neuroanat 26:317-330. CrossRef Medline

Harris MA, Wolbers T (2012) Ageing effects on path integration and landmark navigation. Hippocampus 22:1770-1780. CrossRef Medline

Hartley T, Maguire E, Spiers H, Burgess N (2003) The well-worn route and the path less traveled: distinct neural bases of route following and wayfinding in humans. Neuron 6:877-888. CrossRef Medline

Head D, Isom M (2010) Age effects on wayfinding and route learning skills. Behav Brain Res 209:49-58. CrossRef Medline

Iaria G, Palermo L, Committeri G, Barton JJ (2009) Age differences in the formation and use of cognitive maps. Behav Brain Res 196:187-191. CrossRef Medline

Kessels RP, van Doormaal A, Janzen G (2011) Landmark recognition in Alzheimer's dementia: spared implicit memory for objects relevant for navigation. PLoS One 6:e18611. CrossRef Medline

Kim JH, Son YD, Kim HK, Lee SY, Cho SE, Kim YB, Cho ZH (2011) Effects of age on dopamine D2 receptor availability in striatal subdivisions: a high-resolution positron emission tomography study. Eur Neuropsychopharmacol 21:885-891. CrossRef Medline

King JA, Burgess N, Hartley T, Vargha-Khadem F, O’Keefe J (2002) Human hippocampus and viewpoint dependence in spatial memory. Hippocampus 12:811-820. CrossRef Medline

Kray J, Lindenberger U (2000) Adult age differences in task switching. Psychol Aging 15:126-147. CrossRef Medline

Lipman PD (1991) Age and exposure differences in acquisition of route information. Psychol Aging 6:128-133. CrossRef Medline

Lister JP, Barnes CA (2009) Neurobiological changes in the hippocampus during normative aging. Arch Neurol 66:829-833. CrossRef Medline

Luis CA, Keegan AP, Mullan M (2009) Cross validation of the Montreal Cognitive Assessment in community dwelling older adults residing in the Southeastern US. Int J Geriatr Psychiatry 24:197-201. CrossRef Medline

McDonald RJ, White NM (1994) Parallel information processing in the water maze: evidence for independent memory systems involving dorsal striatum and hippocampus. Behav Neural Biol 61:260-270. CrossRef Medline

McGeorge AJ, Faull RL (1989) The organization of the projection from the 
cerebral cortex to the striatum in the rat. Neuroscience 29:503-537. CrossRef Medline

Moffat SD, Resnick SM (2002) Effects of age on virtual environment place navigation and allocentric cognitive mapping. Behav Neurosci 116:851859. CrossRef Medline

Moffat SD, Kennedy KM, Rodrigue KM, Raz N (2007) Extrahippocampal contributions to age differences in human spatial navigation. Cereb Cortex 17:1274-1282. CrossRef Medline

Nakano K, Kayahara T, Tsutsumi T, Ushiro H (2000) Neural circuits and functional organization of the striatum. J Neurol 247:V1-V15. CrossRef Medline

Nasreddine ZS, Phillips NA, Bédirian V, Charbonneau S, Whitehead V, Collin I, Cummings JL, Chertkow H (2005) The Montreal Cognitive Assessment, MoCA: a brief screening tool for mild cognitive impairment. J Am Geriatr Soc 53:695-699. CrossRef Medline

Nicolle MM, Prescott S, Bizon JL (2003) Emergence of a cue strategy preference on the water maze task in aged C57B6 x SJL F1 hybrid mice. Learn Mem 10:520-524. CrossRef Medline

Packard MG, McGaugh JL (1996) Inactivation of hippocampus or caudate nucleus with lidocaine differentially affects expression of place and response learning. Neurobiol Learn Mem 65:65-72. CrossRef Medline

Raz N, Lindenberger U, Rodrigue KM, Kennedy KM, Head D, Williamson A, Dahle C, Gerstorf D, Acker JD (2005) Regional brain changes in aging healthy adults: general trends, individual differences and modifiers. Cereb Cortex 15:1676-1689. CrossRef Medline

Rodgers MK, Sindone JA, Moffat SD (2012) Effects of age on navigation strategy. Neurobiol Aging 33:15-22. CrossRef Medline

Stranahan AM, Mattson MP (2010) Selective vulnerability of neurons in layer II of the entorhinal cortex during aging and Alzheimer's disease. Neural Plast 2010:108190. CrossRef Medline

Tupala E, Hall H, Bergström K, Mantere T, Räsänen P, Särkioja T, Hiltunen J, Tiihonen J (2003) Different effect of age on dopamine transporters in the dorsal and ventral striatum of controls and alcoholics. Synapse 48:205-211. CrossRef Medline

Voermans NC, Petersson KM, Daudey L, Weber B, Van Spaendonck KP, Kremer HP, Fernández G (2004) Interaction between the human hippocampus and the caudate nucleus during route recognition. Neuron 43:427-435. CrossRef Medline

Voorn P, Vanderschuren LJ, Groenewegen HJ, Robbins TW, Pennartz CM (2004) Putting a spin on the dorsal-ventral divide of the striatum. Trends Neurosci 27:468-474. CrossRef Medline

Waller D, Lippa Y (2007) Landmarks as beacons and associative cues: their role in route learning. Mem Cognit 35:910-924. CrossRef Medline

Wiener JM, Kmecova H, de Condappa O (2012) Route repetition and route retracing: effects of cognitive aging. Front Aging Neurosci 4:7. CrossRef Medline

Wolbers T, Büchel C (2005) Dissociable retrosplenial and hippocampal contributions to successful formation of survey representations. J Neurosci 25:3333-3340. CrossRef Medline

Wolbers T, Weiller C, Büchel C (2004) Neural foundations of emerging route knowledge in complex spatial environments. Cogn Brain Res 21: 401-411. CrossRef Medline

Yankner BA, Lu T, Loerch P (2008) The aging brain. Annu Rev Pathol 3:4166. CrossRef Medline

Yin HH, Knowlton BJ (2004) Contributions of striatal subregions to place and response learning. Learn Mem 11:459-463. CrossRef Medline 